\title{
Editorial
}

\section{Patients in cyberspace: information or confusion?}

Providing health information has been the preserve of health professionals for many years. Patients seeking information about medical disorders are often restricted to enquiring from health workers, friends, and colleagues, or joining self-help groups, which are often supported by medical professionals. The print and electronic media also serves as an important source of information on illness, disease, and the latest medical research. This medical information is often culled or adapted from prestigious medical journals, ${ }^{1}$ which are effectively inaccessible to the general public.

\section{The Internet}

With the advent of the Internet, medical information is now readily available ${ }^{2}$ to anyone who needs it in 'cyberspace', ${ }^{3}$ the notional environment in which electronic communication occurs. The past few years have seen an increase in Internet facilities in all spheres of life, such as homes, offices, institutions, public libraries and even socially, with the emerging culture of 'Internet cafes'. Some organisations provide free Internet access to the public, and in some areas it has been observed that the patient population has greater access to the Internet than their doctors. ${ }^{4}$

\section{Medical information}

On the Internet, the patient can now search ('surfing the net') and immediately access information on symptoms, signs and disorders from all over the world. ${ }^{5}$ While some of this information may emanate from top-notch institutes of medical research, some may be opinions from private websites. As access to, and publishing on the Internet is largely uncontrolled, the quality of this medical information may be extremely variable. Increasing concern has been expressed about the proliferation of unverified claims about therapies on the Internet and the far-reaching implications for patient care. ${ }^{6}$

Through the Internet, instant publishing and immediate retrieval of medical information, which could not be achieved with even the most modern publishing methods, is now possible. However, unlike the scientific journal, or general medical and specialist medical texts, most of the medical information on the Internet is not subjected to peer review or to any editorial procedure. This poses considerable problems, especially given the current drive for 'evidence-based medicine'. In medicine, the ability to review scientific literature critically, to identify major research flaws, and to interpret correctly the clinical implications of research findings, are skills acquired through training, eg, in journal clubs and research seminars. Even with such training, opinions may be divided. In cyberspace, the increasing availability of medical data to clinicians is likely to be matched by an increase in the clinical information available to patients, who may be ill-equipped to interpret it, or indeed to recognise what is 'evidence based', or from an authoritative source.

On the positive side, provision of accurate and appropriate information on risk factors, symptoms, signs, diagnosis and available treatment options, will put healthcare consumers in a better position to make informed decisions on their care. For instance, patients facing an operation can find out more about the process and discuss it with surgeons, anaesthetists, and intensive care specialists on the Internet. With equal ease, information can be found about, for example, psychosocial support for those with breast cancer, or the side-effects of medications used to treat psychiatric disorders. One must caution, that doctorpatient interactions in cyberspace may have major ethical implications.

Problems can arise as a result of this unlimited access to information, however. For example, patients may access the latest research on their disorders, causing them anxiety, raising expectations, or causing them to insist on therapies which may be inappropriate, unaffordable, unavailable or even unknown to the doctors. This information may be specific to a country, untried, or concern a product still in the research phase. There may be information on treatments which are unlicensed, illegal, or even obsolete.

\section{The challenge}

There are several new challenges arising from this situation. As they become accustomed to easily accessible health education information on the Internet, patients may also expect easy access to different treatments and full consultation from their physicians. Doctors may also find themselves exposed to increased legal risk, ${ }^{7}$ as litigation and negligence claims are made by patients on the basis of guidelines and information obtained from Internet sources.

Patients may find themselves in conflict, confusion, or free-fall in this information galaxy. It is up to the medical profession to help patients and carers in search of medical information to find it without unnecessary suffering. As the public thirst for information reaches unsatiable heights, doctors should be involved in its management by acting as experts, increasing patient awareness, providing general information on diagnosis and treatment, and providing referral resources to patients. ${ }^{8}$ This medical involvement in the creation and use of information systems will create more authoritative sources of clinical information for public access. Recognised professional bodies such as the British Medical Association, Royal Colleges and Specialist Institutes should rise to the challenge by creating news groups,

\section{Learning points}

- medical information is readily available on the Internet anywhere in the world

- the Internet is emerging as a major educational tool for doctors, patients and carers alike

- the quality of information on the Internet is extremely varied

- the informed and/or confused patient may pose a challenge to healthcare provision

- the medical profession needs to keep abreast of developments in information technology and become involved in information management 
guidelines, and on-line information resources for lay consumption as reliable sources of current and appropriate information.

\section{The future}

As Internet use widens, spanning geopolitical boundaries, cultures, and socio-economic status, medical practice will change. Emphasis will be placed on our public health role

1 Entwistle V. Reporting research in medical journals and newspapers. $B M \mathcal{F}$ 1995;310:920-3.

2 Kiley R. Medical information on the Internet. Edinburgh: Churchill 2 Kiley R. Medical

3 Thompson D. The concise Oxford dictionary of current english. New York: Oxford University Press, 1995.

4 Fridsma DB, Ford P, Altman R. A survey of patient access to electronic mail: attitudes, barriers and opportunities. Proceedings of the Symposium on Computer Applications in Medicine. $\mathcal{F} \mathrm{Am}$ Med Informat Assoc 1994;(suppl):15-9. as educators, correcting or interpreting information and acquainting patients with authoritative sources of health information in cyberspace - as long as we are not confused ourselves.

OYEDEII AYONRINDE

Department of Forensic Psychiatry, Three Bridges Regional Secure Unit, Middlesex UB1 3EU, UK

Accepted 10 March 1998

5 Nally M. Patient power in the net. Nursing Times 1996;92:31

6 Bower H. Internet sees growth of unverified health claims. BMf 1996;313: 6 Bow.

7 Coiera E. The Internet's challenge to health care provision. BMF 1996;312: $3-4$.

8 Huang MP, Alessi NE. The Internet and the future of psychiatry. Am $\mathcal{f} P s y$ chiatry 1996;153:861-9. 